\title{
Current Understanding of van der Waals Effects in Realistic Materials
}

\author{
Alexandre Tkatchenko \\ Fritz-Haber-Institut der Max-Planck-Gesellschaft, \\ Faradayweg 4-6, 14195 Berlin, Germany*
}

(Dated: October 23, 2014)

\begin{abstract}
Van der Waals (vdW) interactions arise from correlated electronic fluctuations in matter and are therefore present in all materials. Our understanding of these relatively weak yet ubiquitous quantum mechanical interactions has improved significantly during the last decade. This understanding has been largely driven by the development of efficient methods that now enable the modeling of vdW interactions in many realistic materials of interest for fundamental scientific questions and technological applications. In this work, we briefly review the physics behind the currently available vdW methods, highlighting their applications to a wide variety of materials, ranging from molecular assemblies to solids with and without defects, nanostructures of varying size and dimensionality, as well as interfaces between inorganic and organic materials. The origin of collective vdW interactions in materials is discussed using the concept of topological dipole waves. We focus on the important observation that the full many-body treatment of vdW interactions becomes crucial in the investigation and characterization of materials with increasing complexity, especially when studying their response properties, including vibrational, mechanical, and optical phenomena. Despite significant recent advances, many challenges still remain in the development of accurate and efficient methods for treating vdW interactions that will be broadly applicable to the modeling of functional materials at all relevant length and time scales.
\end{abstract}

*tkatchenko@fhi-berlin.mpg.de 


\section{INTRODUCTION}

Functional materials are becoming increasingly smaller in size and more heterogeneous in composition. These two aspects of novel nano-materials lead to the emergence of non-trivial quantum mechanical effects that depend on size and topology, and which may ultimately determine the properties of a material of interest. One important consequence of this evolution beyond traditional materials, whose functionality was largely regulated by bulk observables, is that non-covalent interactions will play an increasingly more crucial role in determining the structure, stability, and ensuing function of homogeneous and heterogeneous nanostructured materials.

In particular, van der Waals (vdW) interactions, which arise from correlated fluctuations of electrons in matter $[1,2]$ and can exhibit non-trivial scaling behavior with system size [3-5], are often the dominant part of such non-covalent interactions. In general, vdW interactions have already been recognized as playing an instrumental role in determining the structure, stability, and functionality of biological materials, supramolecular and sensor chemistry, pharmaceuticals, dye-sensitized solar cells, and many other systems. More recently, the field of "van der Waals heterostructures" [6] has moved into the forefront, and has already lead to fundamental advances in the study of low-dimensional materials and to a number of novel technological applications.

In this context, the importance of understanding and accurately modeling vdW interactions in realistic materials can hardly be overemphasized. However, our ability to model these ubiquitous quantum mechanical effects has been severely impeded by the prohibitively high computational cost of explicitly correlated quantum chemical methods and the lack of efficient approximations to the many-electron correlation problem for large systems [7]. In fact, most successful approximations employed for modeling vdW interactions in materials rely on the rather crude lowest-order pairwise additive (or proximity force) approximation, which is only exact in the weak polarization limit and at large interatomic distances. For condensed-phase systems with a moderate to large polarizability density, such pairwise approximations can often lead to qualitatively incorrect predictions of structural, energetic, and response properties [3-5, 8-11].

The purpose of this article is to provide a succint review of some of the recent progress made in the development of accurate and efficient methods for the treatment of vdW in- 
teractions in realistic materials. As such, the concise nature of this work simply does not allow for a comprehensive review of all of the available methods for describing vdW interactions, hence we refer the reader to Refs. [12-16] and references therein for such a purpose. Rather, the focus herein will be on showcasing the role of vdW interactions in determining the properties of homogeneous and heterogeneous materials of interest in different subfields

of materials science. As the materials universe is vast, we will concentrate on molecular clusters and solids, hard solids with and without defects, nanostructures of varying size and dimensionality, and finally interfaces between inorganic and organic materials. Throughout this article, we will not only demonstrate the significance of vdW interactions in each of these classes of materials, but we will also highlight some of the novel insights that can be gained by utilizing an accurate and fundamental treatment of vdW interactions that is based on quantum mechanics.

\section{EXACT AND APPROXIMATE TREATMENTS OF VDW INTERACTIONS}

The exact energy obtained via the solution of the many-electron Schrödinger equation for a realistic system would seamlessly include the vdW contribution to the energy as well. However, explicitly solving the Schrödinger equation for more than a few electrons is still a prohibitive, if not impossible, task to date, even when certain approximations are employed. Therefore, first principles modeling of realistic materials often starts with coarse-grained mean-field models, such as the Hartree-Fock approximation (HFA), or the alternative suite of density-functional approximations (DFAs), which focus on the three-dimensional electron charge density, $n(\mathbf{r})$ in lieu of the more complicated many-body wavefunction. Unfortunately, these commonly utilized approximations are unable to describe the long-range electronic correlation energy and therefore fail to treat vdW interactions.

However, an exact expression for the electron correlation energy, $E_{c}$, can be constructed by invoking the adiabatic connection fluctuation-dissipation theorem (ACFDT) $[17,18]$

$$
E_{c}=-\frac{\hbar}{2 \pi} \int_{0}^{\infty} d \omega \int_{0}^{1} d \lambda \operatorname{Tr}\left[\left(\chi_{\lambda}\left(\mathbf{r}, \mathbf{r}^{\prime}, \mathrm{i} \omega\right)-\chi_{0}\left(\mathbf{r}, \mathbf{r}^{\prime}, \mathrm{i} \omega\right)\right) v\left(\mathbf{r}, \mathbf{r}^{\prime}\right)\right]
$$

In this expression, $\chi_{0}\left(\mathbf{r}, \mathbf{r}^{\prime}, \mathrm{i} \omega\right)$ is the bare, or non-interacting, response function $\left(\mathbf{r}\right.$ and $\mathbf{r}^{\prime}$ refer to spatial electronic coordinates and $\omega$ is the frequency of the electric field $), \chi_{\lambda}\left(\mathbf{r}, \mathbf{r}^{\prime}, i \omega\right)$ is the interacting response function at Coulomb coupling strength $\lambda, v\left(\mathbf{r}, \mathbf{r}^{\prime}\right)=\left|\mathbf{r}-\mathbf{r}^{\prime}\right|^{-1}$ 
is the Coulomb potential, and $\operatorname{Tr}$ denotes the spatial trace operator (or six-dimensional integration) over the variables $\mathbf{r}$ and $\mathbf{r}^{\prime}$. The essential physical idea behind the ACFDT expression in Eq. 1 is an interpolation between a reference non-interacting mean-field system with $\lambda=0$ and the fully interacting many-body system with $\lambda=1[17,18]$.

The ACFDT approach offers a tractable expression for $E_{c}$ (including the contributions arising from $\mathrm{vdW}$ interactions) provided that a set of single-particle orbitals computed with DFAs (or even the HFA) can be used to construct $\chi_{0}\left(\mathbf{r}, \mathbf{r}^{\prime}, \mathrm{i} \omega\right)$ in Eq. 1. In this regard, the construction of $\chi_{0}\left(\mathbf{r}, \mathbf{r}^{\prime}, \mathrm{i} \omega\right)$ still remains a formidable computational task for systems with thousands of electrons. Furthermore, approximations are still necessary to determine $\chi_{\lambda}\left(\mathbf{r}, \mathbf{r}^{\prime}\right.$, i $\left.\omega\right)$ for $0<\lambda \leq 1$ in Eq. 1 . During the last decade, the so-called random-phase approximation (RPA), which provides a simple recipe for computing $\chi_{\lambda}\left(\mathbf{r}, \mathbf{r}^{\prime}, \mathrm{i} \omega\right)$ has been successfully implemented in many molecular and solid-state electronic structure codes [1922]. Very encouraging results in terms of accuracy have been reported for a multitude of simple materials when $\chi_{0}\left(\mathbf{r}, \mathbf{r}^{\prime}, \mathrm{i} \omega\right)$ is constructed within the RPA using DFA-based orbitals [19-22]. As such, the computational exploration of current RPA methods as well as the theoretical development of improved RPA methods constitute a prominent research area in the electronic structure of realistic materials. Despite this fact, ACFDT-RPA calculations that utilize all of the electronic degrees of freedom are still rather computationally expensive and are currently restricted to static energy evaluations on materials with approximately one hundred atoms per unit cell. This computational bottleneck of ACFDT-RPA is likely to persist for some time and therefore the development of more efficient approximations for computing $E_{c}$ in Eq. 1 is necessary.

The power and significance of the ACFDT approach is that essentially all existing methods for modeling vdW interactions can be derived from approximations to Eq. 1. For example, the widely employed pairwise approximation is obtained by truncating the ACFDT expression to second order in the perturbative expansion of the Coulomb interaction. The simple addition of interatomic vdW potentials that is used to compute the vdW energy in classical force fields and DFA calculations can be recovered from Eq. 1 by further approximating the response function as a sum of independent dipole oscillators located at every nucleus in a given material [23]. The vdW-DF approach originated by Langreth, Lundqvist, and collaborators [24-26] that has become widely used to correct semi-local DFAs can also be derived from Eq. 1 by making a local approximation to the response function in terms of the 
electron density and then employing second-order perturbation theory. However, the main shortcoming of all these rather efficient approximations is that they are unable to capture the non-trivial many-body effects contained in the interacting response function $\chi_{\lambda}\left(\mathbf{r}, \mathbf{r}^{\prime}, \mathrm{i} \omega\right)$ as well as the infinite-order nature of the ACFDT expression in Eq. 1. The inclusion of manybody effects requires going beyond the commonly employed second-order approximation and this can be accomplished by introducing a model system of atomic response functions, which is the topic of the next section.

\section{ATOMIC RESPONSE FUNCTIONS AND DIPOLE WAVES}

The construction of the bare response function, $\chi_{0}\left(\mathbf{r}, \mathbf{r}^{\prime}, \mathrm{i} \omega\right)$ in Eq. 1, from single-particle orbitals is one of the major computational bottlenecks when calculating the electronic correlation energy via the ACFDT expression. Here, we stress that $\chi_{0}\left(\mathbf{r}, \mathbf{r}^{\prime}, i \omega\right)$ contains information about the response of a given molecular system at all interelectronic distances, including short $\left|\mathbf{r}-\mathbf{r}^{\prime}\right|$ separations. However, state-of-the-art DFA methods can often quite successfully treat such short-range electron correlation effects by employing semi-local functionals of the electron density; therefore, the main issue is to construct reliable approximations for the long-range vdW correlations in the context of density functional theory. When describing such long-range, or non-local, electron correlation, the full electronic information contained in $\chi_{0}\left(\mathbf{r}, \mathbf{r}^{\prime}, \mathrm{i} \omega\right)$ is often unnecessary. This is especially the case when one is treating non-metallic materials (and even materials with weakly metallic states), as it is possible to describe the bare response of these systems by a set of localized atomic response functions (ARFs). In this regard, ARFs can be constructed to accurately capture the electronic response beyond a certain cutoff distance, i.e., for $\left|\mathbf{r}-\mathbf{r}^{\prime}\right|>R_{c}$.

The ARF concept has been employed for several decades now in efficient methods for determining molecular polarizabilities and computing the optical spectra of materials. ARFs have also been used to compute vdW interactions in model systems starting nearly 50 years ago [27-31]. However, only recently has the concept of ARFs been extended to enable calculations of the non-local vdW correlation energy in a wide variety of realistic materials [15], by employing spatially-extended ARFs that increase the applicability of the ARF model to include close contact (i.e., for distances as short as the chemical bond) [32, 33].

The ARF model system can also be utilized to gain insight into the emergence of collective 
many-body phenomena that comprise vdW interactions. To illustrate this point, let us start with a material that is built up of a single element (i.e., carbon nanostructures). Each atom $p$ in this material will be represented by a single dipole oscillator with a frequency-dependent polarizability (FDP) given by

$$
\alpha_{p}(\mathrm{i} \omega)=\frac{\alpha_{0} \omega_{0}^{2}}{\omega_{0}^{2}+\omega^{2}},
$$

where $\alpha_{0}$ is the static polarizability and $\omega_{0}$ is an effective excitation (or resonant) frequency. Then the bare response (or bare ARF) for this atom can be written by condensing the response of the valence electrons into a single atom-centered dipole oscillator at position $\mathbf{R}_{p}$ via

$$
\chi_{0, p}\left(\mathbf{r}, \mathbf{r}^{\prime}, \mathrm{i} \omega\right)=-\alpha_{p}(\mathrm{i} \omega) \nabla_{\mathbf{r}} \delta^{3}\left(\mathbf{r}-\mathbf{R}_{p}\right) \otimes \nabla_{\mathbf{r}^{\prime}} \delta^{3}\left(\mathbf{r}^{\prime}-\mathbf{R}_{p}\right) .
$$

The bare response function for a collection of atoms follows as simply the direct sum over the individual ARFs, $\chi_{0}\left(\mathbf{r}, \mathbf{r}^{\prime}, \mathrm{i} \omega\right)=\chi_{0, p}\left(\mathbf{r}, \mathbf{r}^{\prime}, \mathrm{i} \omega\right) \oplus \chi_{0, q}\left(\mathbf{r}, \mathbf{r}^{\prime}, \mathrm{i} \omega\right) \oplus \cdots$.

Collective many-body vdW effects arise because of dynamical correlations between electrons (or ARFs) due to the underlying Coulomb interaction, the effects of which are contained in the interacting response function $\chi_{\lambda}\left(\mathbf{r}, \mathbf{r}^{\prime}, i \omega\right)$. For a system of ARFs in the dipole approximation, these interactions renormalize the values of the bare polarizabilities $\alpha_{0}$ and frequencies $\omega_{0}$ of the isolated ARFs. By allowing a systems of $N$ ARFs to interact, one obtains a set of $3 N$ eigenvectors (dipole waves), expanded in the basis of ARF coordinates. Each dipole wave $i$ arises from a characteristic collective fluctuation of these coupled ARFs and is associated with a renormalized frequency, $\omega_{1, i}$, and static polarizability, $\alpha_{1, i}$. Because of charge conservation, the oscillator strength of every dipole wave, $\alpha_{1, i} \omega_{1, i}^{2}$, must be equal to $\alpha_{0} \omega_{0}^{2}$. Let us assume that the frequency of one of the collective eigenmodes, $\omega_{1, i}$, is equal to $\omega_{0} / 2$. Then, charge conservation implies that $\alpha_{1, i}=4 \alpha_{0}$. It is well-known that the strength of the vdW interaction depends on the magnitude of the vdW coefficients, for example the leading-order vdW $C_{6}$ coefficient scales as $\alpha_{1, i}^{2} \omega_{1, i}$ [34]. Substituting $\alpha_{1, i}$ and $\omega_{1, i}$ in this expression demonstrates that the vdW coefficient of the $i$-th dipole wave is increased by a factor of 8 compared to the bare atoms. This leads to a large modification of the vdW energy due to the frequency renormalization induced by dynamic electron correlations (see Figure 1 and discussion in the next section). In fact, the vdW interaction between dipole waves $i$ and $j$ does not necessarily scale as as sum over interatomic $C_{6} / R^{6}$ terms, and at any finite separation, this could potentially even lead to a modification of the interaction power 
laws, rendering the simple pairwise picture obsolete.

The degree of renormalization of $\omega_{1, i}$ depends sensitively on the topology and dimensionality of the material of interest. Stronger renormalization effects are typically found in low-dimensional systems with high bare polarizability densities. Our understanding of collective effects in vdW interactions is still incomplete and it is clear that more interesting observations are yet to be made. The idea of renormalization discussed above applies to materials of any size and dimensionality, but the most interesting vdW effects are expected in heterogeneous nanostructured materials. We illustrate this with an investigation of carbon nanostructures in the following section.

We note in passing that in the preceding discussion we employed the dipole approximation for the ARFs. This is not a mandatory approximation and one can utilize the full response function, with the ARFs mutually coupled by the Coulomb potential [35]. This approach would also capture higher-order multipole effects and non-linear polarization, and the importance of these effects for long-range vdW interactions in materials still remains to be thoroughly assessed.

\section{DIPOLE WAVES IN CARBON NANOSTRUCTURES}

Perhaps the best example of the emergence of delocalized dipole waves is exhibited by carbon nanomaterials. One can systematically vary the dimensionality, topology, and size of carbon nanostructures, ranging from fullerenes, one-dimensional nanotubes, two-dimensional graphene, to three-dimensional graphite and diamond. It is often assumed that one can model vdW interactions in carbon nanomaterials by associating a $C_{6}$ coefficient to every carbon atom that depends exclusively on the hybridization state of that atom. Different hybridization states (i.e., $\mathrm{sp}, \mathrm{sp}^{2}, \mathrm{sp}^{3}$ ) have $C_{6}$ coefficients that vary on the order of 10-20\% [34, 36]. However, this simple definition misses the important many-body effects beyond trivial hybridization or coordination. To correctly account for these effects, we must calculate the interacting response function $\chi_{\lambda}\left(\mathbf{r}, \mathbf{r}^{\prime}, i \omega\right)$ with $\lambda=1$. The interacting $C_{6}$ coefficients obtained from $\chi_{1}$ for a system of ARFs modeling realistic carbon nanostructures are shown in Figure 1. For a detailed discussion of the response and vdW interactions in carbon nanostructures we refer the reader to Ref. [5]. Here it is sufficient to point out that the carbon-carbon $C_{6}$ coefficients can vary by up to an order of magnitude depending on 
the dimensionality and topology, ranging from roughly 18 hartree.bohr ${ }^{6}$ in small fullerenes to 150 hartree $\cdot \mathrm{bohr}^{6}$ in graphene. The large increase of the $C_{6}$ coefficient in graphene stems from an effective coupling in 2D between the initially localized ARFs. This leads to marked anisotropy in the dielectric response of graphene, yielding dipole waves that are strongly delocalized in the plane of the graphene sheet and characterized by a large polarizability. The same reasoning explains the variations observed in the $C_{6}$ coefficients in carbon nanotubes and graphene nanoribbons as a function of their chirality or size. The rich dielectric properties of nanomaterials as manifested by different $C_{6}$ coefficients could lead to novel self-assembly behavior as predicted in Ref. [5].

\section{THE MBD METHOD FOR VDW INTERACTIONS}

The fact that the electronic response of realistic systems can be fairly delocalized strongly supports the need to develop explicit many-body methods for vdW interactions with the aim to achieve a reliable description of functional materials. To move beyond the traditionally employed second-order pairwise additive approximations, our group has recently developed the so-called MBD (many-body dispersion) method [15, 32, 33]. The essential idea of the MBD method is to map the response of a given nucleoelectronic system into an auxiliary system of coupled ARFs, by using the Tkatchenko-Scheffler (TS) prescription to determine the parameters of the ARFs from the electron density [34]. In the TS method, the vdW parameters are functionals of the electron density $n(\mathbf{r})$, hence they respond to changes in the electron density induced by hybridization, static charge transfer, and other electron redistribution processes.

For ARFs coupled by a dipole potential, the ACFDT expression in Eq. 1 can be computed in a numerically exact way with a computational cost scaling as $N^{3}$, by diagonalizing the interaction Hamiltonian for a system of $N$ ARFs. The algorithm utilized for MBD calculations is shown in a very condensed form in Figure 2. So far, MBD calculations have been applied to systems with up to tens of thousands of atoms per unit cell, but larger calculations are certainly possible with efficient implementations. The correlation energy computed with the MBD method is only a small, albeit fundamental, part of the total electronic energy. To calculate the total energy, the MBD energy must be coupled with DFA calculations that include short-range electron correlation as well as other relevant components of the total 


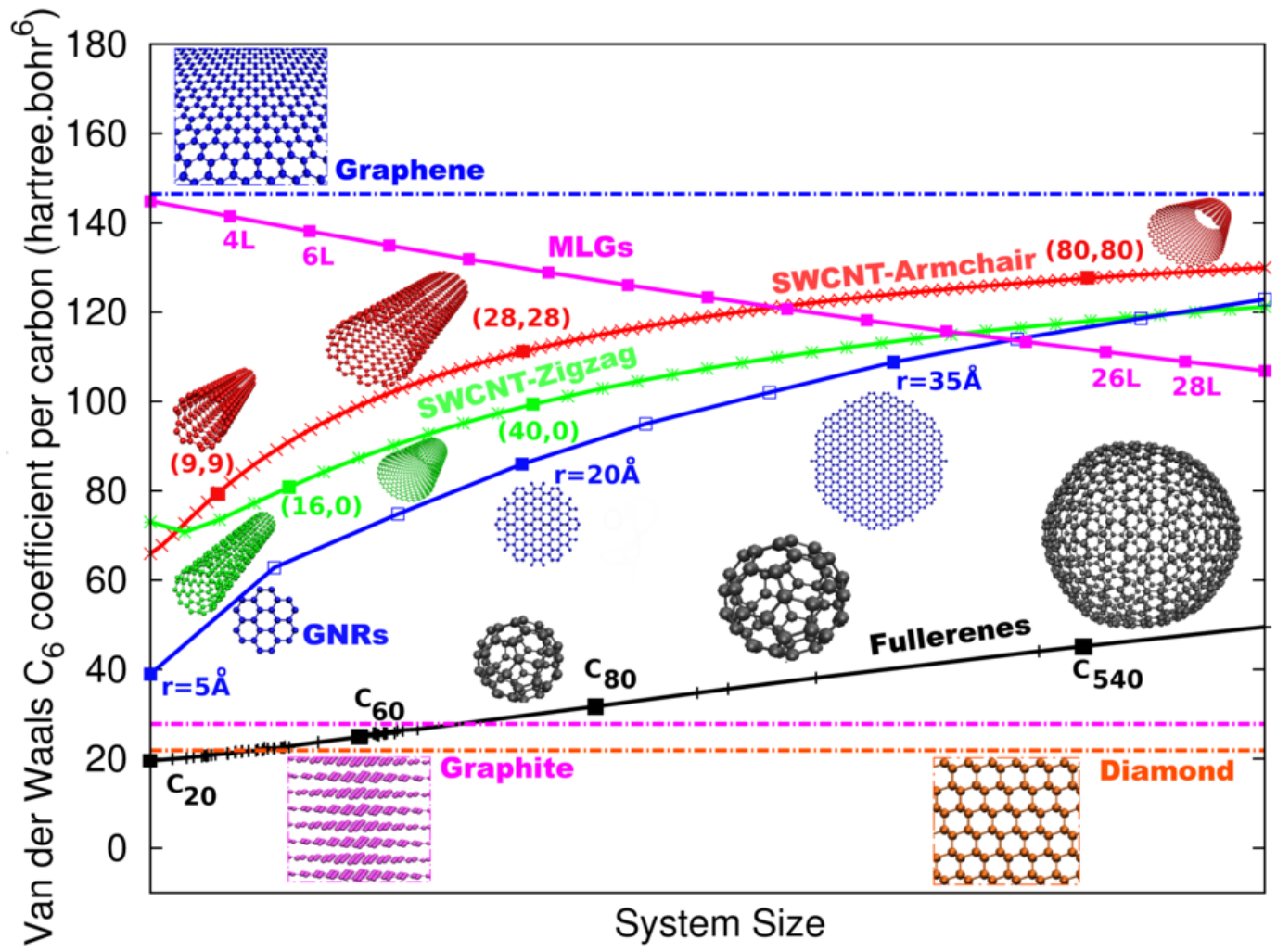

FIG. 1. Van der Waals $C_{6}$ coefficients per carbon atom (the $C_{6}$ of the full system divided by $N_{C}^{2}$, where $N_{C}$ is the number of carbon atoms) for nanostructures of different dimensionality, as calculated for a system of interacting ARFs [15, 32]. The size ranges for the different systems are: (1) The radius of fullerenes is varied from 2 to $12 \AA ;(2)$ The radius of single-wall carbon nanotubes, (SWCNT)-Armchair(n,n) and SWCNT-Zigzag(n,0), vary between 2 and $60 \AA$; (3) The graphene nanoribbons (GNRs) vary in radius from 5 to $50 \AA$; (4) The number of layers in multilayer graphene (MLG) varies from 2 to 30, where each point on the plot corresponds to an increase of 2 layers. Figure adapted from Ref. [5].

energy [37]. When modeling materials, one needs to choose non-empirical DFAs that can be universally applied to both molecules and solids. The widely used PBE functional [38] and hybrid functionals built upon the PBE prescription (PBE0 [39, 40] and HSE [41]) are among the best choices in this regard.

It is important to note that the MBD method relies on several crucial approximations, which must be kept in mind when assessing the reliability of MBD calculations for realistic materials. First, the mapping of electronic orbitals to ARFs is not unique, however our comprehesive investigations for more than 7000 molecules and 50 solids show that the MBD 

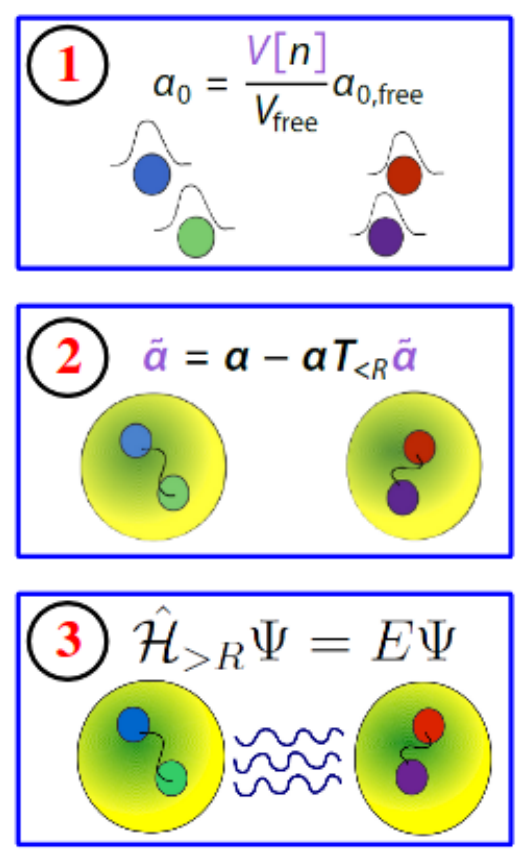

FIG. 2. Schematic diagram illustrating the computation of the long-range vdW correlation energy via the MBD method. The first step is to map the response of realistic material to a system of ARFs by using its electron density. The second step is to account for short-range many-body screening effects that are crucial in condensed materials by solving the Dyson-like response equation. The final step is to couple the ARFs by a long-range dipole potential and solve the interacting Schrödinger equation exactly, which is equivalent to solving Eq. 1. See Ref. [33] for further details.

mapping can successfully reproduce the response properties of systems with finite electronic gaps, including, for example, many organic molecules and even narrow-gap semiconductors such as Ge. However, metallic systems with delocalized single-particle excitations represent a challenge for the current implementation of MBD. Extended molecules with extensively delocalized orbitals or complex resonance structures might also pose a problem for the mapping of the problem to ARFs. Currently, we are assessing an extension of the MBD method for systems containing delocalized single-particle excitations [42]. The second approximation that makes the MBD method particularly efficient is the bilinear dipole potential that is used for coupling the ARFs, instead of the full non-linear Coulomb potential. Since the MBD method is used to compute the non-local long-range correlation energy, the dipole approximation should be reliable. However, in systems with strong polarization one might expect non-linear polarization effects beyond the dipole approximation to become relevant. 
Finally, the coupling between a given DFA and MBD requires the usage of a semi-empirical long-range potential, which has known limiting behavior, but a non-unique functional form. To scrutinize the effects of all these approximations, we have applied the MBD method in conjuction with several DFAs to a wide variety of systems. Several representative applications are briefly summarized in the next section of this manuscript.

\section{APPLICATIONS}

In this section, we highlight some applications of the DFA+MBD method (or approximations thereof) to a wide variety of materials, ranging from molecular assemblies to solids with and without defects, and interfaces between inorganic and organic materials. An important observation to note here is that the full many-body treatment of vdW interactions becomes critical in the investigation of materials of increasing complexity, especially when studying their response properties, including vibrational, mechanical, and optical phenomena. In particular, we will not only demonstrate the significance of $\mathrm{vdW}$ interactions in each of these classes of materials, but we will also highlight some of the novel insights that can be gained by utilizing an accurate and fundamental treatment of vdW interactions that is based on quantum mechanics.

\section{A. Soft organic materials}

The structure and binding in organic materials is often driven by vdW interactions, in combination with other contributions such as repulsion, electrostatics, and induction. There-

fore, accurate modeling of $\mathrm{vdW}$ interactions is critical for understanding the properties of organic materials. In recent years, substantial progress has been achieved in the theoretical prediction of structures and stabilities of molecular crystals by using vdW-inclusive DFA approaches $[43,44]$. Today, the structures of (simple) organic molecular crystals can be predicted with an accuracy of 2-3\% [45-47] and cohesive energies to $1-2 \mathrm{kcal} / \mathrm{mol}[43-45,48]$. However, even for soft organic materials, many-body correlation effects can become significant, and their inclusion can lead to novel qualitative behavior. In Figure 3 we show the performance of DFA+MBD calculations compared to DFA+TS results, which is a pairwise vdW-inclusive method. The inclusion of many-body correlation effects in vdW interac- 
tions clearly leads to a significant increase in accuracy compared to a pairwise method. The effects of many-body interactions become more visible for larger and increasingly inhomogeneous systems. In fact, the polarization of supramolecular complexes and molecular crystals depends sensitively on the geometric structure and their effective dimensionality. This leads to emergence of topological dipole waves as explained in previous sections. Such effects are correctly captured by the MBD method, but are completely missing in simple pairwise approaches to $\mathrm{vdW}$ interactions. It is worth mentioning that empirical parameterization of pairwise methods can be carried out to yield better performance than shown for the DFA+TS method in Figure $3[43,44]$. However, such parameterization may not be tranferable to systems outside of the training set. Explicit many-body methods, such as $\mathrm{DFA}+\mathrm{MBD}$, can capture subtle electronic correlation effects and, therefore, they are substantially more transferable to heterogeneous molecular systems of interest to materials scientists.

Many-body vdW correlations become even more relevant when one looks at the relative energetics of molecular systems, which are essential to predict the polymorphic behavior of molecular crystals. We have demonstrated that only upon including MBD effects one is able to correctly reproduce the structures and relative stabilities of polymorphs of three different molecules: glycine, oxalic acid and tetrolic acid [9]. In fact, DFA+MBD was able to achieve an accuracy of $0.2 \mathrm{kcal} / \mathrm{mol}$ in the relative energetics of glycine polymorphs - an accuracy not achieved so far by any other available method. Another interesting example of a polymorphic system is the aspirin crystal, where there has been a long-standing controversy about the relative stability of two different polymorphs, so-called form I and form II [49]. We have recently demonstrated that the stability of the most abudant form I of aspirin crystal arises from an unexpected coupling between collective vibrational and electronic degrees of freedom (dynamic plasmon-phonon coupling) [11]. In this case, many-body vdW correlations renormalize phonon frequencies of the form I of aspirin, leading to low-frequency phonon modes that increase the entropy of form I and ultimately determine the stability of this ubiquitous form of aspirin in comparison to the metastable form II. Furthermore, the bulk moduli and the shear moduli of both forms of aspirin are substantially modified and become in better agreement with experimnents with DFA+MBD when compared to DFA+TS calculations. The aspirin example illustrates how the inclusion of many-body $\mathrm{vdW}$ effects may lead to novel qualitative predictions for the polymorphism and elastic 


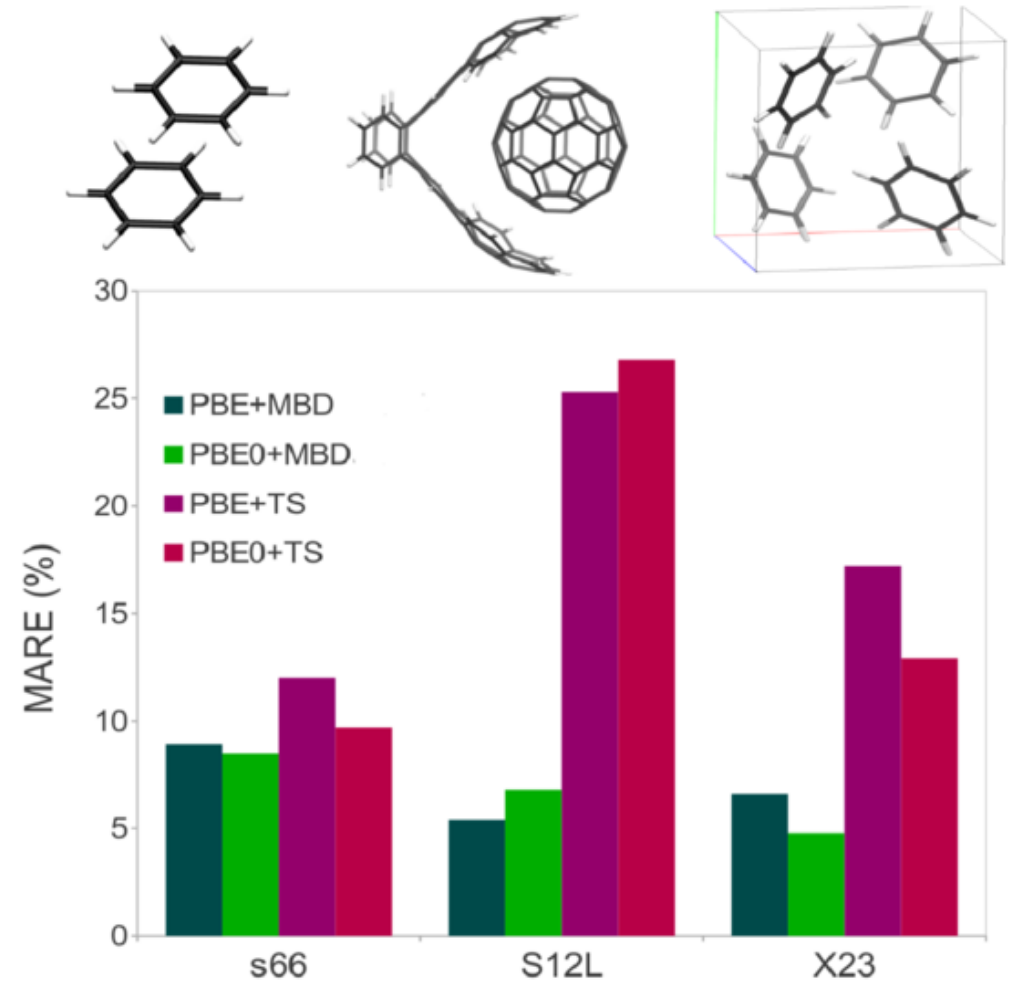

FIG. 3. Mean absolute relative error (MARE, in \%) for intermolecular binding energies in several databases: S66 (molecular dimers), S12L (large supramolecular complexes) and X23 (molecular crystals) computed with MBD and TS methods combined with both PBE and PBE0 non-empirical DFAs. The reference binding energies were calculated using high-level correlated methods for S66 and S12L databases, and extrapolated from experimental sublimation enthalpies for the X23 database. See Ref. [33] for more details.

response of molecular materials.

Another clear manifestation of many-body correlation effects is the sensitive dependence of the optical spectra and dielectric properties of molecular systems on their shape and size or upon formation of a molecular crystal. For example the visible color of oligoacene crystals changes from transparent in naphthalene and anthracene, to bright orange in tetracene, and deep blue in pentacene $[50,51]$. The optical absorption spectrum is directly related to the polarizability through the Kramers-Kronig transformation. Therefore, the observed changes in the optical spectrum upon crystallization of polyacenes are accompanied by a change in the molecular polarizability. These changes in polarization will also directly impact the crystal lattice energy [52]. 


\section{B. Hard solids with and without defects}

While the crucial role of vdW interactions in organic materials is well established, our understanding of the relative importance of these ubiquitous interactions in semiconductors, ionic solids, and metals, is still in development. It has been demonstrated that the contribution of the long-range vdW energy to the cohesive energy of elemental and binary semiconductors and ionic solids amounts to $0.2-0.3 \mathrm{eV} /$ atom [53], around $8 \%$ of the cohesive energy. The contribution of vdW energy to the bulk modulus is even more pronounced, reaching up to $22 \%$ for Ge and GaAs. Notably, the inclusion of vdW interactions in DFA calculations allows to simultaneously improve the performance for lattice constants, cohesive energies, and bulk moduli, when compared to experiment [53]. Similar conclusions have been reached for a wide variety of hard solids when using the so-called opt-vdWDF functionals [54]. Because vdW interactions typically have larger contributions to relative energetics than absolute ones, one expects significant effects for phase transition pressures and phase diagrams of most solids.

The properties of many materials are substantially affected by the presence of simple and complex defects. For example, the properties of semiconductors are largely determined by neutral and charged interstitials and vacancies [55]. The formation of defects entails a modification of polarization around defect sites and this can have a substantial effect on the contribution of vdW energy to the stability and mobility of defects. We have demonstrated that the inclusion of many-body vdW interactions in DFA improves the description of defect formation energies, significantly changes the barrier geometries for defect diffusion, and brings migration barrier heights into close agreement with experimental values [56]. In the case of Si, the vdW energy substantially decreases the migration barriers of interstitials and impurities by up to $0.4 \mathrm{eV}$, qualitatively changing the diffusion mechanism [56]. Recently, the accuracy of our calculations has been confirmed by explicit ACFDT-RPA calculations [20].

Moving beyond point defects, it is to be expected that more complex neutral and charged multi-atom defects and dislocations will lead to even stronger non-local polarization effects and intricate dependence of vdW interactions on the nature of defects. This remains an interesting avenue to explore in future work. 


\section{Interfaces between inorganic and organic materials}

Hybrid inorganic/organic systems (HIOS) are relevant for many applications in catalysis, light-emitting diodes, single-molecule junctions, molecular sensors and switches, and photovoltaics. Obviously, the predictive modeling and understanding of the structure and stability of such hybrid systems is an essential prerequisite for tuning their electronic properties and functions. The bonding in HIOS is often determined by a delicate balance between covalent bonds, hydrogen bonds, charge transfer, Pauli repulsion, and vdW interactions. [57] Indeed, until recent developments for efficiently incorporating the long-range vdW energy within state-of-the-art DFAs, it was not possible to study the structure and stability of realistic HIOS.

The difficulty in applying DFA+MBD to HIOS is that the inorganic substrate is often a metal or a doped semiconductor. For such systems, the projection to initially-localized ARFs used in the MBD method might be too restrictive to correctly describe metallic single-particle excitations. To address this issue, we have developed an approximation to DFA+MBD, called DFA+vdW ${ }^{\text {surf }}[58]$. This method fully includes the many-body screening effects within the extended substrate, but treats the molecule-substrate interaction using the pairwise approximation.

We have applied the DFA+vdW $\mathrm{W}^{\text {surf }}$ method to a wide variety of molecules adsorbed on metallic substrates [59]. In these applications, we were able to achieve quantitative accuracy for aromatic hydrocarbons (benzene, naphthalene, anthracene, and diindenoperylene), $\mathrm{C}_{60}$, and sulfur/oxygen-containing molecules (thiophene, NTCDA, and PTCDA) on close-packed and stepped metal surfaces, leading to an overall accuracy of $0.1 \AA$ in adsorption heights and $0.1 \mathrm{eV}$ in binding energies with respect to state-of-the-art experiments. An unexpected finding is that vdW interactions contribute more to the binding of strongly bound molecules on transition-metal surfaces than for molecules physisorbed on coinage metals [60]. The accurate inclusion of vdW interactions also significantly improves tilting angles and adsorption heights for all the studied molecules, and can qualitatively change the potential-energy surface for adsorbed molecules with flexible functional groups. Activation barriers for molecular switches and reaction precursors are modified as well [61].

Ongoing work concentrates on understanding the interplay between many-body effects within the inorganic bulk material with collective effects within the adsorbed molecular lay- 
ers. Initial studies indicate that DFA+MBD can be successfully applied to treat adsorption on semiconductors and transition metals, and that the adsorption energies and monolayer formation energies obtained with DFA+MBD are in even better agreement with experiments than when using the more approximate DFA+vdW ${ }^{\text {surf }}$ method.

Finally, we note that the MBD method can also be used to predict the structures and dielectric properties of model dye-sensitized solar cells and other hybrid structures [62, 63]; the obtained information can be used for example to calculate the band gap renormalization of condensed hybrid materials.

\section{PERSPECTIVES AND FUTURE CHALLENGES}

The development of increasingly sophisticated methods for vdW interactions during the last decade has allowed to achieve many novel insights into the structural, energetic, and response properties of realistic materials. Yet, many significant challenges remain on the path towards fully predictive modeling of these ubiquitous quantum-mechanical interactions and fully understand their role in the properties of functional materials. Among the many contemporary challenges, we would like to highlight the following:

1. Developing increasingly more accurate and broadly applicable methods for heterogeneous materials including those with highly delocalized electronic states. This requires systematically investigating the reliability of the ARF approximation and possibly extending it for materials with small band gaps.

2. Understanding the emergence of topological dipole waves in heterogeneous materials and interfaces between materials with vastly different dielectric properties. Could we engineer interaction power laws and control the self-assembly of interfacial structures based on "unconventional" delocalized vdW correlations?

3. Scaling the applicability of methods to increasingly larger system sizes, eventually bridging microscopic methods that correctly treat the non-locality of electronic response with mesoscopic methods that treat vdW/Casimir physics and include effects of finite speed of light (retardation) and finite temperature on vdW/Casimir interactions. 
4. Traditionally, it is assumed that vdW interactions have a negligible direct contribution in modifying the electronic and optical properties of materials. Our recent self-consistent implementation of the DFA+TS method indicates that vdW interactions can directly affect the electronic properties of surfaces and HIOS. For example, workfunctions of coinage metal surfaces can be modified by as much as $0.3 \mathrm{eV}$, because vdW interactions tend to increase surface dipole moments. However, our understanding of this issue is still in its infancy. Much more work is required to assess the relative importance of vdW interactions for static and dynamic electronic and optical properties.

\section{BIOGRAPHY}

Alexandre Tkatchenko obtained his Ph.D. in physical chemistry in 2007 at the Universidad Autonoma Metropolitana, Mexico. During 2008-2010, he was an Alexander von Humboldt Fellow in the Theory Department at FHI, Berlin. Currently, he leads a group on Functional Materials and Intermolecular Interactions at FHI, partially funded through a Starting Grant from the European Research Council. He has received the Gerhard Ertl Young Investigator Award of the German Physical Society (2011).

\section{ACKNOWLEDGMENTS}

This work is supported by the European Research Council (ERC-StG VDW-CMAT). Dr. Robert A. DiStasio Jr. is gratefully acknowledged for inspiring discussions. 
[1] Langbein, D. Theory of Van der Waals attraction; Springer-Verlag, 1974; Vol. 72.

[2] Parsegian, V. A. Van der Waals forces: A Handbook for Biologists, Chemists, Engineers and Physicists; Cambridge University Press, 2005.

[3] Dobson, J. F.; White, A.; Rubio, A. Phys. Rev. Lett. 2006, 96, 073201.

[4] Ruzsinszky, A.; Perdew, J. P.; Tao, J.; Csonka, G. I.; Pitarke, J. M. Phys. Rev. Lett. 2012, 109, 233203.

[5] Gobre, V. V.; Tkatchenko, A. Nat. Commun. 2013, 4, 2341.

[6] Geim, A. K.; Grigorieva, I. V. Nature 2013, 499, 419.

[7] Szabo, A.; Ostlund, N. S. Modern quantum chemistry: Introduction to advanced electronic structure theory; Dover Publications: New York, 1996.

[8] Tkatchenko, A.; O. A. von Lilienfeld,; R. A. DiStasio Jr., Proc. Natl. Acad. Sci. USA 2012, 109, 14791.

[9] Marom, N.; DiStasio Jr., R. A.; Atalla, V.; Levchenko, S.; Reilly, A. M.; Chelikowsky, J. R.; Leiserowitz, L.; Tkatchenko, A. Angew. Chem., Int. Ed. 2013, 52, 6629-6632.

[10] Ambrosetti, A.; Alfè, D.; R. A. DiStasio Jr.,; Tkatchenko, A. J. Phys. Chem. Lett. 2014, 5, 849-855.

[11] Reilly, A. M.; Tkatchenko, A. Phys. Rev. Lett. 2014, 113, 055701.

[12] R. French et al., Rev. Mod. Phys. 2010, 82, 1887.

[13] Klimeš, J.; Michaelides, A. J. Chem. Phys. 2012, 137, 120901.

[14] Dobson, J. F.; Gould, T. J. Phys.: Condens. Matter 2012, 24, 073201.

[15] R. A. DiStasio Jr.,; Gobre, V. V.; Tkatchenko, A. J. Phys.: Condens. Matter 2014, 26, 213202.

[16] Grimme, S.; Antony, J.; Ehrlich, S.; Krieg, H. J. Chem. Phys. 2010, 132, 154104.

[17] Gunnarsson, O.; Lundqvist, B. I. Phys. Rev. B 1976, 13, 4274.

[18] Langreth, D. C.; Perdew, J. P. Phys. Rev. B 1977, 15, 2884.

[19] Harl, J.; Kresse, G. Phys. Rev. B 2008, 7r7, 045136.

[20] Kaltak, M.; Klimeš, J.; Kresse, G. Phys. Rev. B 2014, 90, 054115.

[21] Eshuis, H.; Furche, F. J. Phys. Chem. Lett. 2011, 2, 983.

[22] Ren, X.; Rinke, P.; Joas, C.; Scheffler, M. J Mater. Sci. 2012, 47, 7447. 
[23] Tkatchenko, A.; Ambrosetti, A.; R. A. DiStasio, Jr., J. Chem. Phys. 2013, 138, 074106.

[24] Dion, M.; Rydberg, H.; Schroder, E.; Langreth, D. C.; Lundqvist, B. I. Phys. Rev. Lett. 2004, 92, 246401.

[25] Lee, K.; Murray, E. D.; Kong, L.; Lundqvist, B. I.; Langreth, D. C. Phys. Rev. B 2010, 92, 081101.

[26] Cooper, V. R.; Kong, L.; Langreth, D. C. Physics Procedia 2010, 3, 1417.

[27] Bade, W. L. J. Chem. Phys. 1957, 27, 1280.

[28] Donchev, A. G. J. Chem. Phys. 2006, 125, 074713.

[29] Cole, M. W.; Velegol, D.; Kim, H.-Y.; Lucas, A. A. Mol. Simul. 2009, 35, 849.

[30] Shtogun, Y. V.; Woods, L. M. J. Phys. Chem. Lett. 2010, 1, 1356.

[31] Liu, R.-F.; Angyan, J. G.; Dobson, J. F. J. Chem. Phys. 2011, 134, 114106.

[32] Tkatchenko, A.; R. A. DiStasio, Jr.,; Car, R.; Scheffler, M. Phys. Rev. Lett. 2012, 108, 236402.

[33] Ambrosetti, A.; Reilly, A. M.; DiStasio Jr., R. A.; Tkatchenko, A. J. Chem. Phys. 2014, 140, $18 \mathrm{~A} 508$.

[34] Tkatchenko, A.; Scheffler, M. Phys. Rev. Lett. 2009, 102, 073005.

[35] Jones, A. P.; Crain, J.; Sokhan, V. P.; Whitfield, T. W.; Martyna, G. J. Phys. Rev. B 2013, 87, 144103.

[36] Wu, Q.; Yang, W. J. Chem. Phys. 2002, 116, 515.

[37] Burke, K. J. Chem. Phys. 2012, 136, 150901.

[38] Perdew, J. P.; Burke, K.; Ernzerhof, M. Phys. Rev. Lett. 1996, 7r, 3865.

[39] Perdew, J. P.; Ernzerhof, M.; Burke, K. J. Chem. Phys. 1996, 105, 9982.

[40] Adamo, C.; Barone, V. J. Chem. Phys. 1999, 110, 6158.

[41] Heyd, J.; Scuseria, G. E.; Ernzerhof, M. J. Chem. Phys. 2003, 118, 8207.

[42] Hermann, J.; Scheffler, M.; Tkatchenko, A. in preparation.

[43] Otero-de-la-Roza, A.; Johnson, E. R. J. Chem. Phys. 2012, 137, 054103.

[44] Brandenburg, J. G.; Grimme, S. Top. Curr. Chem. 2014, 345, 1-23.

[45] Reilly, A. M.; Tkatchenko, A. J. Chem. Phys. 2013, 139, 024705.

[46] Schatschneider, B.; Monaco, S.; Tkatchenko, A.; ; Liang, J. J. Phys. Chem. A 2013, 117, 8323-8331.

[47] Schatschneider, B.; Monaco, S.; Liang, J.-J.; Tkatchenko, A. J. Phys. Chem. C 2014, 118, 19964. 
[48] Reilly, A. M.; Tkatchenko, A. J. Phys. Chem. Lett. 2013, 4, 1028-1033.

[49] Ouvrard, C.; Price, S. L. Cryst. Growth Des. 2004, 4, 1119-1127.

[50] Pope, M.; Swenberg, C. E. Electronic Processes in Organic Crystals and Polymers; Oxford University Press, New York, 1999.

[51] Anthony, J. E. Angew. Chem. Int. Ed. 2008, 47, 452.

[52] Schatschneider, B.; Liang, J.-J.; Reilly, A. M.; Marom, N.; Zhang, G.-X.; Tkatchenko, A. Phys. Rev. B 2013, 87, 060104.

[53] Zhang, G.-X.; Tkatchenko, A.; Paier, J.; Appel, H.; Scheffler, M. Phys. Rev. Lett. 2011, 107, 245501.

[54] Klimeš, J.; Bowler, D. R.; Michaelides, A. Phys. Rev. B 2011, 83, 195131.

[55] Freysoldt, C.; Grabowski, B.; Hickel, T.; Neugebauer, J.; Kresse, G.; Janotti, A.; Van de Walle, C. G. Rev. Mod. Phys. 2014, 86, 253-305.

[56] Gao, W.; Tkatchenko, A. Phys. Rev. Lett. 2013, 111, 045501.

[57] Tkatchenko, A.; Romaner, L.; Hofmann, O. T.; Zojer, E.; Ambrosch-Draxl, C.; Scheffler, M. MRS Bull. 2010, 35, 435.

[58] Ruiz, V. G.; Liu, W.; Zojer, E.; Scheffler, M.; Tkatchenko, A. Phys. Rev. Lett. 2012, 108, 146103.

[59] Liu, W.; Tkatchenko, A.; Scheffler, M. Modeling Adsorption and Reactions of Organic Molecules at Metal Surfaces, Acc. Chem. Res. (2014), doi: 10.1021/ar500118y.

[60] Liu, W.; Carrasco, J.; Santra, B.; Michaelides, A.; Scheffler, M.; Tkatchenko, A. Phys. Rev. B 2012, 86, 245405.

[61] Liu, W.; Filimonov, S. N.; Carrasco, J.; Tkatchenko, A. Nat. Commun. 2013, 4, 2569.

[62] Marom, N.; Moussa, J. E.; Ren, X.; Tkatchenko, A.; Chelikowsky, J. R. Phys. Rev. B 2011, $84,245115$.

[63] Marom, N.; Korzdorfer, T.; Ren, X.; Tkatchenko, A.; Chelikowsky, J. R. J. Phys. Chem. Lett. 2014, 5, 2395 . 